\title{
Lockdown-associated Hunger May Be Affecting Breastfeeding: Findings From a Large SMS Survey in South Africa
}

Nazeeia Sayed ( $\square$ nazeeia.sayed@gmail.com )

University of the Western Cape https://orcid.org/0000-0003-4629-5230

Ronelle Burger

University of Stellenbosch

Abigail Harper

University of the Witwatersrand

Elizabeth C (Rina) Swart

University of the Western Cape

\section{Short report}

Keywords: breastfeeding, hunger, food security

Posted Date: January 13th, 2021

DOI: https://doi.org/10.21203/rs.3.rs-141928/v1

License: @ (1) This work is licensed under a Creative Commons Attribution 4.0 International License. Read Full License

Version of Record: A version of this preprint was published at International Journal of Environmental Research and Public Health on December 30th, 2021. See the published version at https://doi.org/10.3390/ijerph19010351. 


\section{Abstract}

There are growing concerns on the impact of COVID-19 on food security and child health in South Africa. A rapid SMS Maternal and Child Health survey was conducted in South Africa in June $(n=3140)$ with a follow up in July $(n=2287)$. Breastfeeding practices found in this survey confirmed findings from other studies: with high breastfeeding initiation rates and early introduction of other foods/mixed milk feeding. Logistic regression was conducted to explore the associations between breastfeeding, maternal depression and hunger in the household. The odds of hungry mothers breastfeeding were significantly lower (OR $=0.66 ; 95 \% \mathrm{Cl}: 0.44,0.99)$. The prevalence of depression in this survey sample was $26.95 \%$, but there was no association between breastfeeding behaviour and depression scores (OR $=0.89 ; 95 \% \mathrm{Cl}: 0.63,1.27)$. A positive correlation was found between not breastfeeding and not going to the health clinic. Breastfeeding needs to be protected, promoted and supported; and mothers should be encouraged to continue to attend the health clinic. Further to communication to communities, supporting the breastfeeding mother to enable her to breastfeed and feel competent to do so is a critical gap that needs to be addressed. This support could include economic support to enable her to access a nutritious diet. Mothers also need reassurance on the quality of their breastmilk and their ability to breastfeed. Aside from the nutritional superiority, the safety and immune benefits of breastmilk may need to be asserted to counter concerns during the COVID-19 pandemic.

\section{Background}

Improvement of breastfeeding rates remains a key strategy to ensure food security for the first 6 months of life [1, 2]. Although the mechanisms require better understanding, there is a growing body of literature on lower breastfeeding rates and earlier cessation of exclusive breastfeeding with increased severity of food insecurity [3-6]. With the advent of COVID-19 and a national lockdown in South Africa in April and May 2020, food security concerns alongside anticipated negative impact on the fragile, but recently improved breastfeeding rates [7], as well as child development, emerged.

\section{A national mobile survey conducted in 2020}

The data used for this assessment comes from a rapid SMS (Short Message Service) Maternal and Child health (MATCH) survey. This was a national survey conducted among pregnant women and mothers registered with the MomConnect mhealth platform in South Africa. It is estimated that this platform has more than half of all women attending the public sector antenatal care services enrolled [8]. Permission for the survey was obtained from the National Department of Health, and ethics approval was obtained from the University of Stellenbosch's Research Ethics Committee for Social, Behavioural and Education Research [project 14926 on 15 June 2020].

A self-weighted sample of 15000 pregnant women and mothers with children under 12 months was drawn from the database of MomConnect users. The sample was stratified based on province, gestational age or age of their baby and their type of phone. The women received an invitation to join the SMS survey on the afternoon of 24 June 2020. They could respond with "JOIN" to participate, "STOP" to not participate or "MORE" if they needed further information. Those who participated in the survey received R10 in airtime. A response rate of $21 \%(n=3140)$ was achieved for the survey, which ended on 30 June 2020 . A follow-up survey invitation with further questions was sent on 2 July and this survey ended on 5 July 2020. Of the 3140 individuals that responded to the first survey, 2287 also responded to the follow-up survey.

We conducted logistic regression to explore the associations between breastfeeding, maternal depression and hunger in the household. The correlation between breastfeeding and clinic attendance was also explored. Stata 15.2 was used for the analysis.

Depression was calculated using a modified version of the PHQ-2 tool [9]. This likert-like scale asked two questions about the prevalence of depression symptoms in the past week: "In the last 7 days have you felt hopeless, down or depressed? In the last 7 days have you felt little interest or pleasure in doing things?" Respondents then went on to indicate the regularity with which they experienced negative feelings: "no", "yes, a few days", or "yes, most days". Respondents were then assigned a continuous score between 0 and 6 with each increased unit indicating an increased severity of symptoms. For analysis purposes, we used a cut-off of 2 to indicate the likelihood of depressive symptoms.

Questions on hunger in wave 1 enquired if a child in the household had gone hungry in the last seven nights and whether an adult in the household had gone hungry in the last seven nights. In the follow-up survey, the hunger questions focused on whether the respondent had gone hungry in the last seven nights as well as if a child had gone hungry in the past seven nights. We used the guidelines for interpretation of the household hunger scale by Ballard et al. (2011) [10] and scaled it accordingly for a reference period of 7 days. Thus a frequency of once in 7 days was classified as "rarely"; 2 times in 7 days was classified as "sometimes" (equivalent to 3-10 times in 30 
days) and 3 or more times in 7 days was classified as "often" (equivalent to > 10 times in 30 days. "Rarely" was recategorised as food secure after combining cases with a "never" response/at risk of food insecurity; "sometimes" was classified as food insecure and "often" was classified as severe food insecurity.

Breastfeeding information was collected in the second wave (July 2020). Currently breastfeeding was determined by a positive response to the question "Yesterday, did you breastfeed your baby?". Mixed feeding was determined by a positive response to "Yesterday did you feed your baby formula or porridge such as Nestum?" If the answer to the first question was yes, and no to the second question, then a follow up question was posed to identify exclusive breastfeeding by asking if only breastmilk was given, or if formula or infant foods were given in the seven days prior. These three questions were combined to create an indicator for exclusive breastfeeding: categorising women as exclusively breastfeeding if they breastfed their baby the previous day, but did not feed them formula or porridge and also confirmed that over the past seven days they only gave their baby breastmilk (and no formula or infant foods).

The survey included a question asking women to share their main worry. It was an open-ended question and limited only by the 160character limit of SMSes.

\section{Infant feeding practices resonate with previous findings}

We found that amongst mothers with infants of 3 months or younger, $94.1 \%$ said that they breastfed the previous day and $72.2 \%$ were breastfeeding exclusively. It is likely that limitations on movement of people and reduced shopping access, due to a national COVID-19 lockdown, also contributed to more mothers continuing to breastfeed in this sample. By 6 months, $93.1 \%$ of infants were still breastfed, but only $28.6 \%$ were breastfed exclusively. These findings resonate with previous findings in South Africa. Breastfeeding initiation rates in South Africa are high (ranging from 75 to 100\%), but continued breastfeeding varies, and there is widespread early introduction of foods and liquids other than breastmilk/formula milk [11]. The 2016 South African Demographic and Health Survey reported that $32 \%$ of infants younger than six months were exclusively breastfed [12].

Eighteen percent (18.3\%) of our respondents reported going to bed hungry over the past 7 days. Of the respondents who went to bed hungry, $28.2 \%$ rarely went to bed hungry, $37.9 \%$ sometimes went to bed hungry, and $28.0 \%$ often went to bed hungry. One in twenty $(5.9 \%)$ of respondents who went to bed hungry did not want to provide information on how often they went to bed hungry or said they did not know.

The responses to the question on what worried them was stirring. The following quotes are the responses that included comments on hunger, nutrition and breastfeeding:

"I worry about losing my life or my kids due to COVID-19 and not having food in the house, because as a breastfeeding mom I have to eat so that I can produce milk"

"I am concerned about going to bed hungry when I have to breastfeed"

"I am worried about my health and wellbeing together with good nutrition since I am breastfeeding"

While general adult and child hunger in the household were not associated with a decreased prevalence of breastfeeding, women who reported that they went to bed hungry in the past seven nights were significantly less likely to report breastfeeding in the previous day $(O R=0.66 ; 95 \% \mathrm{Cl}: 0.44,0.99)$. Previous studies have also identified a link between food insecurity and breastfeeding. A Canadian study observed that more than half of food insecure mothers had ceased to breastfeed exclusively by 2 months [4]. A longitudinal cohort study in Kenya [13] found that maternal hunger was associated with lower rates of breastfeeding, but that mothers with greater self-efficacy across all levels of hunger were more likely to exclusively breastfeed, than those with poor self-efficacy. Hunger in mothers may contribute to a perception of milk insufficiency and undermine confidence in their breastfeeding ability. A study in Kenya [14] found that there were greater odds that a woman in a food insecure household would consider her breast milk insufficient, and that they would not breastfeed for 6 months. For every 1-point increase in the household food insecurity score, another Kenyan study [15] found that there was a decrease in breastmilk intake by the infant. A 2015 Canadian study postulated that household food insecurity could be a predictor of breastfeeding initiation due to concerns on the cost of alternate feeding, but concerns over their own food and nutrient intake and the quality or quantity of breastmilk they produced, may lead some mothers to introduce formula milk [16]. Reducing hunger in mothers by improving household food security and improving breastfeeding self-efficacy in mothers could lead to higher rates of exclusive breastfeeding $[13,17]$. 


\section{Breastfeeding is not associated with depression}

COVID-19 is likely to increase stress levels in women. The stress that mothers from low-income households in South Africa experience could include concerns on their breastmilk supply, their access to food, having regular meals and relationship difficulties in the home [17]. Women in particular face an increased care burden due to COVID-19 and this further increases the stress on them [18].

The prevalence of depression in this survey sample was $26.9 \%$, but there was no association with breastfeeding behaviour and depression scores $(\mathrm{OR}=0.89 ; 95 \% \mathrm{Cl}: 0.63,1.27)$. Depression could influence a mothers' breastfeeding self-efficacy [19], and may result in a shorter duration of breastfeeding or greater likelihood of breastfeeding cessation $[19,20]$. The bidirectional relationship between depression and breastfeeding may be due to breastfeeding leading to better mother-infant interaction, the release of oxytocin, and decline of cortisol levels, which may improve maternal mood and reduce feelings of stress [21].

\section{Breastfeeding mothers were more likely to not miss their primary health care facility visits}

We found that women who had not gone to the primary health care (PHC) facility recently (in the past two months) were significantly less likely to breastfeed (See Table 1). Eighty-five percent (85\%) of mothers who had been to the PHC facility recently breastfed their baby, while $75 \%$ of those who had not been to the PHC facility recently breastfed their baby. The difference is significant $(p=0.001)$. There is also a significant relationship between exclusive breastfeeding and recent PHC facility attendance. Seventeen percent (17\%) of mothers who had been to the clinic recently exclusively breastfed their babies, while $11 \%$ of those who had not been recently to the clinic exclusively breastfed their babies $(p=0.019)$.

Table 1

Breastfeeding and attendance of PHC facility

\begin{tabular}{|llll|}
\hline \multicolumn{4}{|c|}{ Did you breastfeed your baby yesterday? } \\
\hline Have not been to clinic in 2 months & No & Yes & Total \\
\hline No & $112(15.0 \%)$ & $632(85.0 \%)$ & 744 \\
\hline Yes & $59(25.2 \%)$ & $175(74.8 \%)$ & 234 \\
\hline Total & 171 & 807 & 978 \\
\hline Pearson chi2 $=12.74 \mathrm{Pr}=0.000$ & & & \\
\hline
\end{tabular}

This relationship between clinic visits and breastfeeding could be attributable to the role of primary health care facilities in supporting and encouraging mothers to breastfeed. Alternatively, it could reflect an endogenous relationship between caregiving and care seeking behaviour reflective of intergenerational transmission of attachment [22].

More regular clinic attendance may allow for an increase in knowledge on breastfeeding, improved self-efficacy for breastfeeding, and emotional support to breastfeed [23]. A meta-analysis found that breastfeeding education and support was associated with both breastfeeding initiation and continuation [24]. There are some indications that postnatal support may be slightly more effective than antenatal breastfeeding education [25], but both may be required to impact on breastfeeding continuation [26]. Ultimately, breastfeeding support in a combination of settings (health services, the workplace, the home family and community) along with appropriate policy is required for improved breastfeeding [27].

\section{Limitations}

A key limitation of the findings presented here is the cross-sectional nature of this survey. Hunger and depression have various dimensions that may change with time. The questions and tools used to assess these measures, may also affect the results.

\section{Conclusions}

Based on the findings of this survey that mothers experiencing hunger are less likely to breastfeed, the authors recommend a paradigm shift in efforts to improve breastfeeding, to be more comprehensive. This includes strengthening of enabling capabilities [28] such as health seeking behaviour and self-efficacy; direct nutrition support of pregnant and breastfeeding women; inclusion of the value of 
breastfeeding beyond only an immediate superior nutritional and hygienic feeding method to include the food security and noncommunicable disease prevention aspects; and the inclusion of a whole-of-society approach [29].

Breastfeeding needs to be protected, promoted and supported more than ever as this can impact the health of future generations, and also gives all infants a fairer/more equal start in life. In addition, mothers need to be encouraged to continue to attend the health clinic.

Among the four lifesaving interventions to be prioritised during COVID-19, the UN recommends mass communication to caregivers and families to protect, promote and support breastfeeding in children aged 0-23 months [30]. Further to communication, supporting the breastfeeding mother to enable her to breastfeed and feel competent to do so is a critical gap that needs to be addressed. Lee (2016) [31] argues that the ethical obligation to breastfeed a child extends beyond the mother to society which has to provide social and economic support to enable breastfeeding, and this may also extend to providing food to the hungry breastfeeding mother as a priority (or enabling her to access nutritious food). Maternal and child nutrition needs to be prioritised alongside the COVID-19 response, or we will see the devastating impact of malnutrition, such as stunted growth, diminished cognitive potential and an increased burden of noncommunicable disease in the coming generations [32].

Mothers also need reassurance on the quality of their breastmilk and their ability to breastfeed. From a health perspective, the benefits of breastmilk in giving infants a better start in life is undisputed, and is contrary to hungry mothers stopping breastfeeding due to their concerns and belief that stopping would have a better outcome for their infants. The effect that COVID-19 has had on breastfeeding advice and support in South Africa is not known. South Africa's history and learnings from HIV and infant feeding guidance should not be forgotten and efforts need to include reaffirming the benefits of breastfeeding among our health care workers. Evidence suggests that health care worker infant feeding advice is undermined by implementing national guidelines within local contexts and fears of HIV transmission [33]. Aside from the nutritional superiority, the safety and immune benefits of breastmilk may need to be asserted to counter concerns during the COVID-19 pandemic.

\section{Abbreviations}

SMS: Short Message Service; MATCH: Maternal and Child health; PHC: Primary health care

\section{Declarations}

\section{ETHICS APPROVAL AND CONSENT TO PARTICIPATE}

Ethics approval was obtained from the University of Stellenbosch's Research Ethics Committee for Social, Behavioural and Education Research [project 14926 on 15 June 2020]. Participants consented to participate by responding "JOIN" to the SMS.

\section{CONSENT FOR PUBLICATION}

Not applicable.

\section{AVAILABILITY OF DATA AND MATERIALS}

The datasets analysed during the current study will be available in the DATAFIRST repository, [https://www.datafirst.uct.ac.za/dataportal/index.php/catalog/central/about]

\section{COMPETING INTERESTS}

The authors have no competing interests to declare.

\section{FUNDING}

RB received funding for data collection from The Federated Employers Mutual Assurance Company, Allan and Gill Gray Foundation, as well as the Michael and Susan Dell Foundation.

NS receives support from the DSI/NRF Centre of Excellence in Food Security through a post-doctoral fellowship (project 20302).

\section{AUTHORS' CONTRIBUTIONS}


RB conceptualised the data collection. RB, RS and NS conceptualised the paper. NS did the first draft of the paper. AH, RB and NS did the data analyses. All authors equally contributed to the final paper.

\section{ACKNOWLEDGEMENTS}

We thank those who contributed to the conceptualisation of the instrument design and collection including Peter Barron, Yogan Pillay, Nic Spaull, and Kai Barron. We are grateful for Jane Passmore, Erik Harding, Lesley Bamford, Len Hansen, and Samantha van Schalkwyk for helping to get the permissions and access agreements required.

\section{References}

1. World Health Organization. Global strategy for infant and young child feeding. Geneva, Switzerland: WHO; 2003. [https://apps.who.int/iris/bitstream/handle/10665/42590/9241562218.pdf;jsessionid=7DDC6B6C47D527C48EEAB89B57BED839? sequence=1] Accessed 23 November 2000.

2. Bhutta Z, Ahmed T, Black RE, Cousens S, Dewey K, Giugliani E, ... Shekar M. What works? Interventions for maternal and child undernutrition and survival. Maternal and Child Undernutrition Series. The Lancet. 2008;371,417-40.

3. Venu I, van den Heuvel M, Wong JP, Borkhoff CM, Moodie RG, Ford-Jones EL, Wong MD. The breastfeeding paradox: Relevance for household food insecurity. Paediatrics \& Child Health. 2017;22(4),180-3.

4. Orr SK, Dachner N, Frank L, Tarasuk V. Relation between household food insecurity and breastfeeding in Canada. CMAJ. 2018;190,E312-9.

5. Van den Heuvel M, \& Birken C. Food insecurity and breastfeeding. Canadian Medical Association Journal. 2018;190,E310-1.

6. Dinour LM, Rivera Rodas El, Amutah-Onukagha NN, Doamekpor LA. The role of prenatal food insecurity on breastfeeding behaviors: findings from the United States pregnancy risk assessment monitoring system. International Breastfeeding Journal. 2020;15,30. .

7. Jackson D, Swanevelder S, Doherty T, Lombard C, Bhardwaj S, Goga A. Changes in rates of early exclusive breast feeding in South Africa from 2010 to 2013: data from three national surveys before and during implementation of a change in national breastfeeding policy. BMJ Open. 2019;9, e028095.

8. LeFevre AE, Dane P, Copley CJ, Pienaar C, Parsons AN, Engelhard M, ... Mohan D. Unpacking the performance of a mobile health information messaging program for mothers (MomConnect) in South Africa: evidence on program reach and messaging exposure. BMJ Glob Heal. 2018;3,1-12.

9. Kroenke K, Spitzer R, \& Williams J. The Patient Health Questionnaire-2: Validity of a two-item depression screener. Medical Care. 2003;41(11),1284-92.

10. Ballard T, Coates J, Swindale A, Deitchler M. Household Hunger Scale: Indicator Definition and Measurement Guide. Washington, DC: Food and Nutrition Technical Assistance II Project, FHI 360; 2011.

[https://www.fantaproject.org/sites/default/files/resources/HHS-Indicator-Guide-Aug2011.pdf] Accessed 30 November 2020.

11. Sayed N, Schonfedlt HC. A review of complementary feeding practices in South Africa. South African Journal of Clinical Nutrition. Published online 28 August 2018.

12. National Department of Health, Statistics South Africa, South African Medical Research Council, ICF. South Africa Demographic and Health Survey 2016. Pretoria, South Africa and Rockville, Maryland, USA: NDoH, Stats SA, SAMRC and ICF; 2019.

13. Tuthill EL, Miller JD, Collins SM, Widen EM, Onono M, Young SL. HIV infection, hunger, breastfeeding self-efficacy, and depressive symptoms are associated with exclusive breastfeeding to six months among women in western Kenya: a longitudinal observational study. International Breastfeeding Journal. 2020;15,4.

14. Webb-Girard A, Cherobon A, Mbugua S, Kamau-Mbuthia E, Amin A, Sellen DW. Food insecurity is associated with attitudes towards exclusive breastfeeding among women in urban Kenya. Maternal and Child Nutrition. 2012;8,199-214.

15. Miller JD, Young SL, Boateng GO, Oiye S, Owino V. Greater household food insecurity is associated with lower breast milk intake among infants in western Kenya. Maternal and Child Nutrition. 2019;e12862. _

16. Frank L. Exploring Infant Feeding Practices In Food Insecure Households: What Is The Real Issue? Food and Foodways. 2015;23(3),186-209.

17. Witten C, Claasen N, Kruger HC, Coutsoudis A, Grobler H. Psychosocial barriers and enablers of exclusive breastfeeding: lived experiences of mothers in low-income townships, North West Province, South Africa. International Breastfeeding Journal. $2020 ; 15,76$. 
18. Power K. The COVID-19 pandemic has increased the care burden of women and families, Sustainability: Science, Practice and Policy. 2020;16(1),67-73.

19. Oyetunji A, Chandra P. Postpartum stress and infant outcome: A review of current literature. Psychiatry Research. 2020;284,112769.

20. Dias CC, Figueiredo B. Breastfeeding and depression: A systematic review of the literature. Journal of Affective Disorders. 2015;171,142-154.

21. Pope CJ, Mazmanian D. (2016). Breastfeeding and Postpartum Depression: An Overview and Methodological Recommendations for Future Research. Depression Research and Treatment. 2016;476531. _

22. George C, Solomon J. The caregiving system: A behavioral systems approach to parenting. In: Cassidy J, Shaver P, editors. Handbook of Attachment: Theory, Research, and Clinical Application. New York: Guilford Press; 1999. P. 649-70.

[https://www.researchgate.net/publication/232541328_Attachment_and_caregiving_The_caregiving_behavioral_system] Accessed 27 November 2020.

23. Wood NK, Woods NF, Blackburn ST, Sanders EA. Interventions that enhance breastfeeding Initiation, duration, and exclusivity: A systematic review. The American Journal of Maternal/Child Nursing. 2016;41(5),299-307.

24. Cohen SS, Alexander DD, Krebs NF, Young BE, Cabana MD, Erdmann P, ... Saavedra JM. Factors Associated with Breastfeeding Initiation and Continuation: A Meta-Analysis. The Journal of Pediatrics. 2018;203,190-196.e21.

25. Su L, Chong Y, Chan Y, Chan Y, Doris F, Tun K, ... Rauff M. Antenatal education and postnatal support strategies for improving rates of exclusive breastfeeding: randomised controlled trial. BMJ. 2007;335,596.

26. Schreck PK, Solem K, Wright T, Schulte C, Ronnisch KJ, Szpunar S. Both Prenatal and Postnatal Interventions Are Needed to Improve Breastfeeding Outcomes in a Low-Income Population. Breastfeeding Medicine. 2017;12(3),142-8.

27. Sinha B, Chowdhury R, Sankar MJ, Martines J, Taneja S, Mazumder S, ... Bhandari N. Interventions to improve breastfeeding outcomes: a systematic review and meta-analysis. Acta Paediatr. 2015;104,114-34.

28. Nussbaum M. Creating Capabilities. The Human Development Approach. USA: The Belknap Press of Harvard University Press; 2011.

29. Collins T, Mikkelsen B, Kaarboe O, Walch S, Chestnov O. Investing in healthier cities - multistakeholder action to prevent noncommunicable diseases. Eurohealth. 2017;23(3),9-12.

30. Fore HF, Dongyu Q, Beasley DM, Ghebreyesus TA. Child malnutrition and COVID-19: the time to act is now. The Lancet. Published online 27 July 2020.

31. Lee R. Feeding the Hungry Other: Levinas, Breastfeeding, and the Politics of Hunger, Hypatia. 2016;31(2),259-74.

32. Kent EF. COVID-19's Triple Threat to Gender Equality, Nutrition and Future Potential. 2020. [https://thousanddays.org/updates/covid-19s-triple-threat-to-gender-equality-nutrition-and-future-potential/] Accessed 7 November 2020.

33. Nieuwoudt S, Manderson L. Frontline health workers and exclusive breastfeeding guidelines in an HIV endemic South African community: A qualitative exploration of policy translation. International Breastfeeding Journal. 2018;13,20. 